\title{
Research Article \\ Evaluating Pavement Cracks with Bidimensional Empirical Mode Decomposition
}

\author{
Albert Ayenu-Prah and Nii Attoh-Okine \\ Department of Civil and Environmental Engineering, University of Delaware, Newark, DE 19716-3120, USA
}

Correspondence should be addressed to Nii Attoh-Okine, okine@udel.edu

Received 5 September 2007; Accepted 2 March 2008

Recommended by Daniel Bentil

Crack evaluation is essential for effective classification of pavement cracks. Digital images of pavement cracks have been analyzed using techniques such as fuzzy set theory and neural networks. Bidimensional empirical mode decomposition (BEMD), a new image analysis method recently developed, can potentially be used for pavement crack evaluation. BEMD is an extension of the empirical mode decomposition (EMD), which can decompose nonlinear and nonstationary signals into basis functions called intrinsic mode functions (IMFs). IMFs are monocomponent functions that have well-defined instantaneous frequencies. EMD is a sifting process that is nonparametric and data driven; it does not depend on an a priori basis set. It is able to remove noise from signals without complicated convolution processes. BEMD decomposes an image into two-dimensional IMFs. The present paper explores pavement crack detection using BEMD together with the Sobel edge detector. A number of images are filtered with BEMD to remove noise, and the residual image analyzed with the Sobel edge detector for crack detection. The results are compared with results from the Canny edge detector, which uses a Gaussian filter for image smoothing before performing edge detection. The objective is to qualitatively explore how well BEMD is able to smooth an image for more effective edge detection with the Sobel method.

Copyright (c) 2008 A. Ayenu-Prah and N. Attoh-Okine. This is an open access article distributed under the Creative Commons Attribution License, which permits unrestricted use, distribution, and reproduction in any medium, provided the original work is properly cited.

\section{INTRODUCTION}

Pavement evaluation is an essential part of a good pavement management system for effective maintenance, rehabilitation, and reconstruction (MR\&R) decision making. Pavement evaluation involves condition surveys to monitor the overall health of the pavement network, and recommendations made regarding maintenance actions. Traditionally, pavement condition surveys are visual surveys whereby a crew is sent out to visually inspect sections of pavement for various types of distress. The most popular method is the pavement condition index (PCI) method developed by the United States Army Corps of Engineers. The PCI assessment is a visual procedure by which a selected pavement section is visually evaluated for various distress types, distress severity and quantity. Apart from the method being subjective and depending on the expertise of the inspector, it is also quite expensive. A more objective and less expensive method of distress evaluation is automated pavement distress evalua- tion, which system consists of automatically getting images of distresses and analyzing them using feature selection methods such as edge detection techniques for distress detection and identification. Various image-processing techniques such as fuzzy set theory [1], neural networks [2], and Markov methods [3] have been used to analyze cracking in road pavements. Furthermore, there has been work in the area of aggregate shape characteristics [4-6] using various imaging techniques.

The present paper explores pavement crack detection using a new method called the bidimensional empirical mode decomposition (BEMD) together with a well-known edge detector, the Sobel edge detector. A number of images are smoothed with BEMD to remove noise, and the residual image analyzed with the Sobel edge detector for crack detection. The results are compared with results from the Canny edge detector, which first filters out noise from the image with a Gaussian filter before performing edge detection. The objective is to qualitatively determine how 
well BEMD is able to smooth an image for more effective edge detection using the Sobel method.

\section{BIDIMENSIONAL EMPIRICAL MODE DECOMPOSITION}

The bidimensional empirical mode decomposition (BEMD) is the 2-D extension of the empirical mode decomposition (EMD), which is part of the Hilbert-Huang transform (HHT) developed by Huang et al. [7]. The empirical mode decomposition (EMD) is a multiresolution decomposition method that decomposes signals into basis functions that are adapted from the signals themselves. That is, no a priori basis functions are defined for the decomposition as in Fourier-based methods in which sines and cosines are used as predefined basis functions and then convolved with the signal. Therefore, Fourier methods are most suitable for linear and stationary signals. The EMD is hinged on the idea of instantaneous frequency; instantaneous frequency becomes valid only in the event the signal is made symmetric with respect to the local zero-mean line. Upper and lower envelopes, which cover all local maxima and local minima, respectively, are constructed, and then their mean iteratively removed in order to force local symmetry about the zeromean line; the procedure has been termed "sifting." The sifting process results in the generation of basis functions known as intrinsic mode functions (IMFs), which are adaptively derived from the signal within the local time scale of the signal; IMFs have instantaneous frequency defined for them at every point. Therefore, while the EMD is a local decomposition method, Fourier-based methods are global in nature, which requires a transformation into the frequency domain in order to determine the energy content of the signal; it is not possible to achieve that in the time domain.

The HHT represents the energy content of a signal in an energy-frequency-time domain called the Hilbert spectrum; energy content is analyzed in the time domain so that the exact instance an event occurs is known. It differs from the wavelet transform, however, in that wavelets still need a priori defined basis sets similar to the Fourier transform. Huang et al. [7] gives the full treatment of the HHT method. The process used to generate the Hilbert spectrum is called the Hilbert spectral analysis (HSA). Thus the HHT consists of the two parts, EMD and HSA.

IMFs have certain requirements that need to be met in order to be acceptable:

(i) the number of zero crossings and extrema must be equal or differ by at most one in whole data sets (to remove riding waves); and

(ii) the mean value of the envelope defined by the local maxima and the envelope defined by the local minima must be zero at every point.

An important step in the EMD process is the construction of the maxima and minima envelopes; research has shown that the cubic spline is the best fit for 1-D EMD. There are stopping criteria for the EMD process to prevent the resulting IMFs from being just purely frequency

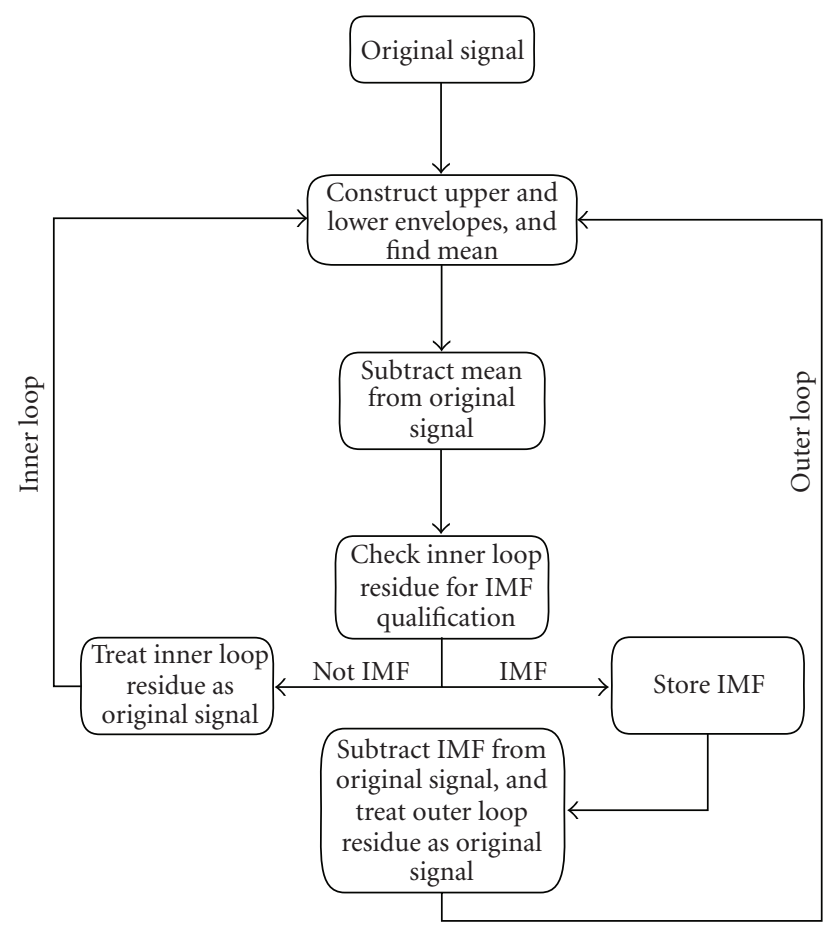

Figure 1: Pictorial representation of EMD.

and amplitude-modulated components. Two stopping criteria have been proposed: a Cauchy-type convergence that depends on limiting the standard deviation computed from two consecutive IMFs [7], and one that depends on the agreement of the numbers of extrema and zero crossings [8]. The whole EMD is stopped when the final residue becomes a monotonic function, or a constant. A snapshot of the sifting process to generate IMFs is shown in Figure 1 in which two loops are presented: the inner loop iterates for IMFs, while the outer loop subtracts the most current IMF from the original signal or what is left of it after previous IMFs have been removed from it, and then passes execution to the inner loop for the next IMF.

The HHT has a number of advantages that make it desirable for signal analysis. The process is empirical and the most computationally intensive step is the EMD operation, which does not involve convolution and other time-consuming operations; this makes HHT ideal for signals of large size. The Hilbert-Huang spectrum does not involve the concept of frequency resolution but instantaneous frequency, which is desirable for local analyses.

The success of the 1-D EMD prompted research into a 2-D version, which may be used for image processing. Linderhed [9] first introduced 2-D EMD, which has been subsequently called bidimensional empirical mode decomposition (BEMD). The basic steps in BEMD are the same as for the EMD, only in two dimensions. Of much importance is the envelope construction for maxima and minima; in this case, scattered data interpolation (SDI) is used to construct 2-D surfaces. Various SDI methods have been used to construct maxima and minima envelopes, 
but unpublished results of recent comprehensive analyses conducted by authors of the present research were not conclusive regarding the superiority of one SDI method over another when various methods were used in BEMD analyses of texture and real images. However, Linderhed [10] preferred radial basis functions (RBFs) with thin-plate splines. The appropriate SDI method would depend on the objective of the BEMD analysis. Before SDI can be performed, appropriate extrema detection needs to be carried out. Detection of extrema has been achieved with methods including morphological reconstruction based on geodesic operators [11], and neighboring windows [10]. The stopping criteria for BEMD are similar to that for the 1-D EMD. BEMD has been used for texture analysis [12] and image compression [13]. Recently, Sinclair and Pegram [14] have used it for rainfall analysis and nowcasting.

\section{EDGE DETECTION}

\subsection{Canny method}

Edges are areas in an image with sharp intensity gradients. The objective of edge detection algorithms is to seek out these points of rapid intensity changes. There are a number of edge detection algorithms, including the Sobel edge detector, the Laplacian of Gaussian method, the Canny edge detector, the fast Fourier transform, the zero-crossing method, the Prewitt method, and the Roberts method. Of all the edge detection algorithms, the Canny edge detector seems to be the most effective in detecting object edges, and the most widely used.

The Canny edge detector detects edges by finding the pixel points where the gradient magnitude is a maximum in the direction of the gradient, that is, in the direction of maximum intensity change. However, the image is first smoothed with a Gaussian filter to remove noise, which is a convolution operation. The detection method is summarized into four steps as follows [15]:

(i) smooth image by convolving with an appropriate Gaussian filter to reduce image details;

(ii) at each pixel, determine gradient magnitude and gradient direction along maximum intensity change;

(iii) mark the pixel as an edge if the gradient magnitude at the pixel is greater than the pixels at both sides of it in the gradient direction;

(iv) remove the weak edges by hysteresis thresholding.

\subsection{Sobel method}

Similar to the Canny method, the Sobel edge detector is also a gradient-based method. It detects edges by searching for maxima and minima in the first derivative of the image. However, the Sobel method does not do any presmoothening of the image; therefore, it is more susceptible to noise, but is computationally less expensive and faster. The Sobel edge detector performs a 2-D spatial gradient calculation on a gray-scale image; two $3 \times 3$ convolution masks are used to calculate gradients, one along the $x$-direction, and the other along the $y$-direction. The masks are given as follows:

$$
\begin{aligned}
& {\left[\begin{array}{ccc}
1 & 2 & 1 \\
0 & 0 & 0 \\
-1 & -2 & -1
\end{array}\right] \text { in the } x \text {-direction; }} \\
& {\left[\begin{array}{ccc}
-1 & 0 & 1 \\
-2 & 0 & 2 \\
-1 & 0 & 1
\end{array}\right] \text { in the } y \text {-direction. }}
\end{aligned}
$$

\subsection{BEMD in edge detection}

The potential application of BEMD is in presmoothing of images before feature detection techniques are applied; this can pave the way for a hybrid method of edge detection that involves the BEMD and an edge detector that does not have a presmoothing step. Images usually tend to be noisy and so filtering out noise is essential to make the image ready for further analysis.

In BEMD, an image is decomposed into basis functions called IMFs; the set of IMFs are complete, so that summing up the IMFs and any residual left recovers the original image. EMD essentially acts as a dyadic filter [16, 17], and by extension, the BEMD also acts as a dyadic filter. It has been observed that the first IMF constitutes most of the noise in the signal [11]. Hence removal of the first IMF reduces high spatial frequencies. Since BEMD is local in nature, image blurring is reduced. Filtering occurs in time space rather than in frequency space; therefore, any nonlinearity and nonstationarity present in the data are preserved. Thus no spurious harmonics are introduced as occurs in traditional Fourier analyses that arise out of a priori definition of sine and cosine basis sets. Although the first IMF has been observed to contain most of the noise, the first few IMFs from BEMD still usually contain a lot of the noise in the original image; therefore, removing them and reconstructing the image with the remaining IMFs tend to denoise the image. The number of IMFs needed to be removed depends on the level of noise in the image; very noisy images require more high-frequency IMFs removed than do less noisy images. The Canny edge detector has a prefiltering step in which images are denoised with a Gaussian filter before edge detection is accomplished. This detection method can be computationally more expensive due to the convolution processes required in Gaussian smoothing. The Sobel edge detection method has no prefiltering step; however, it is more susceptible to noise. Therefore, the BEMD is used to first filter the images before the Sobel method is applied. An advantage BEMD has over Gaussian filtering is that it does not involve any convolution process, and it is a local method of denoising.

Traditional filtering (Gaussian, mean, or median filtering) requires an optimal filter size to perform effectively. However, it is not a trivial matter to determine the optimal filter size; a large filter removes much of the noise but leaves more blur while too small a filter size leaves little blur but may leave a lot of noise. This problem is circumvented by the BEMD because it is a local decomposition technique 
TABLE 1: Detection results for asphalt images.

\begin{tabular}{l|ll}
\hline No. of Images & Canny & BEMD/Sobel \\
\hline \multirow{3}{*}{9} & $\begin{array}{l}\text { Good detection for } 6 \text { of the } 9 \text { images } \\
\text { or } 67 \% \text { of the } 9 \text { images } \\
\text { Of the } 6 \text { images detected, } 4 \text { images } \\
\text { had cracks (representing } 67 \% \text { of the } 6 \text { images) } \\
\text { The remaining } 2 \text { images had no cracks } \\
\text { (representing 33\% of the } 6 \text { images) }\end{array}$ & $\begin{array}{l}\text { Good detection for } 3 \text { of the } 9 \text { images } \\
\text { or 33\% of the } 9 \text { images } \\
\text { Of the } 3 \text { images detected, } 2 \text { images } \\
\text { had cracks (representing 67\% of the } 3 \text { images) } \\
\end{array}$ \\
\hline
\end{tabular}

TABLE 2: Detection results for PCC images.

\begin{tabular}{|c|c|c|}
\hline No. of Images & Canny & BEMD/Sobel \\
\hline \multirow{4}{*}{6} & $\begin{array}{l}\text { Good detection for } 2 \text { of the } 6 \text { images } \\
\text { or } 33 \% \text { of the } 6 \text { images }\end{array}$ & $\begin{array}{l}\text { Good detection for } 2 \text { of the } 6 \text { images } \\
\text { or } 33 \% \text { of the } 6 \text { images }\end{array}$ \\
\hline & \multicolumn{2}{|c|}{$\begin{array}{l}\text { Canny and BEMD/Sobel tied on the remaining } 2 \text { of the } 6 \text { images } \\
\text { (representing } 33 \% \text { of the } 6 \text { images); these images had cracks }\end{array}$} \\
\hline & $\begin{array}{l}\text { Of the } 2 \text { images detected, } 1 \text { image had cracks } \\
\text { (representing } 50 \% \text { of the } 2 \text { images) }\end{array}$ & $\begin{array}{l}\text { Of the } 2 \text { images detected, none had cracks } \\
\text { (representing } 0 \% \text { of the } 2 \text { images) }\end{array}$ \\
\hline & $\begin{array}{l}\text { The remaining } 1 \text { image had no cracks } \\
\text { (representing } 50 \% \text { of the } 2 \text { images) }\end{array}$ & $\begin{array}{l}\text { The remaining } 2 \text { images had no cracks } \\
\text { (representing } 100 \% \text { of the } 2 \text { images) }\end{array}$ \\
\hline
\end{tabular}

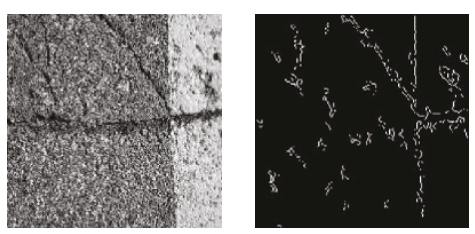

(a) With Canny: asphalt surface

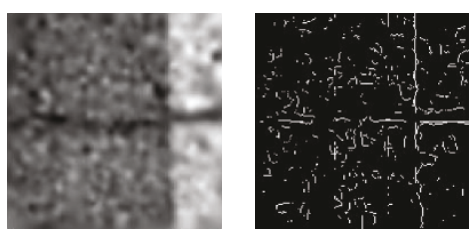

(b) With BEMD/Sobel: asphalt surface

FIgURE 2

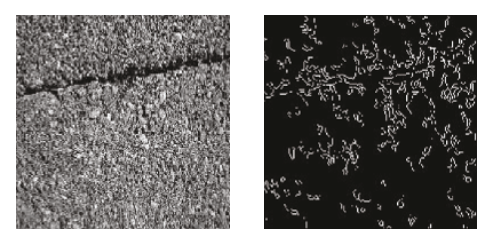

(a) With Canny: asphalt surface

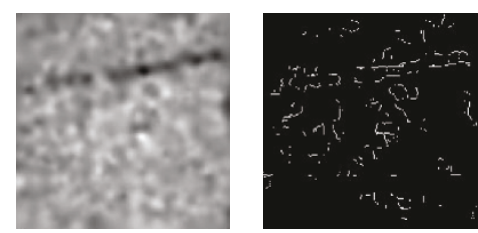

(b) With BEMD/Sobel: asphalt surface

Figure 3 rather than global. For instance, the Gaussian filter incorporates the Fourier transform, which is global and hence introduces some artifacts due to nonstationarity and possible nonlinearity.

\section{ANALYSES}

A total of 15 asphalt concrete and portland cement concrete (PCC) images are analyzed with the Canny edge detector to detect cracks; the same images are again analyzed with the Sobel edge detector, but this time BEMD is first used to smooth the image before detection. The first IMF is removed from the original image and the residue, which is a smoothed image, is analyzed with the Sobel method; the codes used are implemented in Matlab. The objective is to find out if BEMD is able to perform image smoothing for more effective crack detection. There are 9 asphalt concrete images and 6 PCC images. A digital camera was used to take the images in clear weather; each image had a resolution of 256-by-256 pixels. There are images with cracks and images without cracks. For brevity, only 8 images are shown in the present paper: 4 asphalt and 4 PCC images.

Hysteresis thresholding is used to aid in crack detection. The edge detection depends upon selection of appropriate thresholds; improper thresholds may result in many unnecessary edges returned, or insufficient edges that result in 


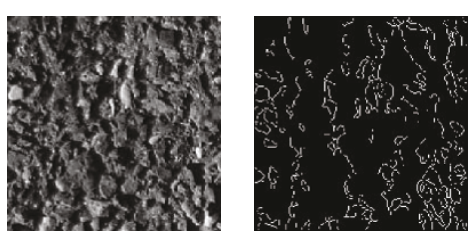

(a) With Canny: asphalt surface
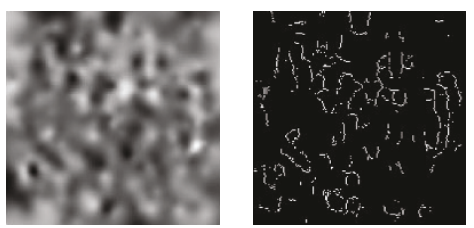

(b) With BEMD/Sobel: asphalt surface

FIGURE 4
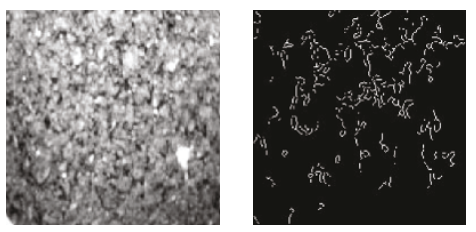

(a) With Canny: asphalt surface
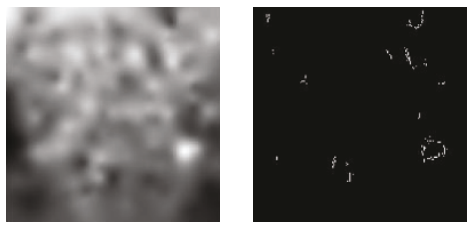

(b) With BEMD/Sobel: asphalt surface

FiguRe 5

missing important edges. A standard deviation is chosen for the Gaussian filter, and the effect of thresholding depends on the chosen standard deviation.

Matlab codes for BEMD are as developed by Nunes et al. [11]; to generate IMFs, upper and lower envelopes are constructed from strict extrema using interpolation by minimum curvature method.

Regarding the images used, asphalt concrete images tend to have a lot of irregularities due to the nature of the finished surface while PCC images tend to be smoother with fewer irregularities. Therefore, detecting cracks on asphalt concrete surfaces can be more challenging than on PCC surfaces.

\section{RESULTS AND DISCUSSION}

Figures 2 to 9 show the results of the edge detection attempts by the Canny edge detector (all the "a" figures above) and by the combination of BEMD and Sobel edge detector method (all the "b" figures below). A summary of the detection results for all 15 images is given in Tables 1 and 2.

After BEMD was performed on an asphalt image, the first three IMFs were discarded. The image was then reconstructed with the remaining IMFs, which was then used as

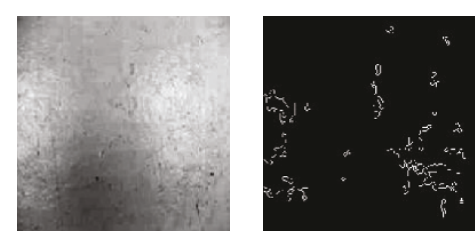

(a) With Canny: PCC surface
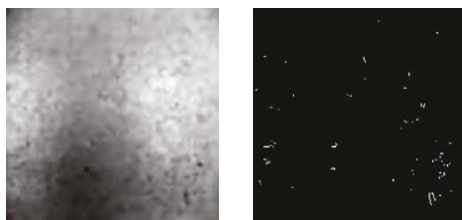

(b) With BEMD/Sobel: PCC surface

FIGURE 6
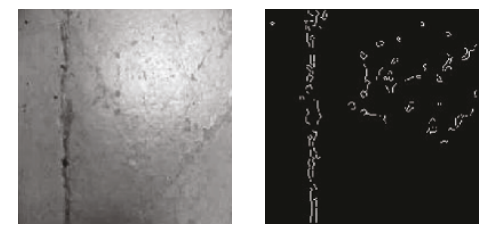

(a) With Canny: PCC surface
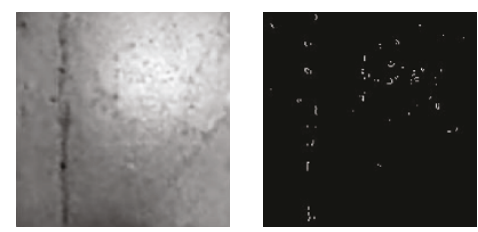

(b) With BEMD/Sobel: PCC surface

Figure 7 the input image for the Sobel Edge Detector. This is necessary after observing that removing only the first IMF does not smooth the image enough for edge detection. However, removal of only the first IMF was sufficient smoothing for the PCC images. The Canny edge detector already has a Gaussian filter, so no BEMD was performed for smoothing.

The Canny edge detector, and the BEMD/Sobel method were able to detect cracks more easily on PCC surfaces, but with a little bit more difficulty for asphalt surfaces. This was expected due to the many irregularities on the asphalt surfaces analyzed. However, the Canny method generally proved better on asphalt surfaces. It is also observed that despite the noisy output of the BEMD/Sobel method, crack edges could be detected on closer examination as may be seen in Figures 2 and 3. In Figure 2, the edge of the lane marking and part of the horizontal crack can be made out in Figure 2(b) despite the noisy output; however, even with less noise, Figure 2(a) (Canny method) is not able to detect the whole length of the horizontal crack, but is able to easily bring out the diagonal crack connecting it at the junction of the lane marking and the horizontal crack. In Figure 3, the crack is more easily identified in Figure 3(b) (BEMD/Sobel). For images with no cracks, as in Figures 4 


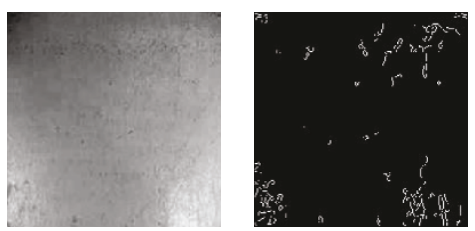

(a) With Canny: PCC surface

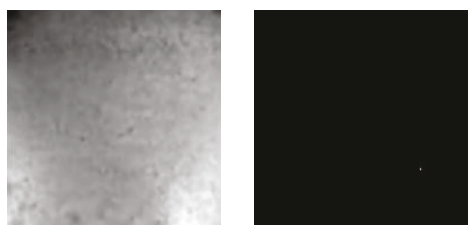

(b) With BEMD/Sobel: PCC surface

Figure 8

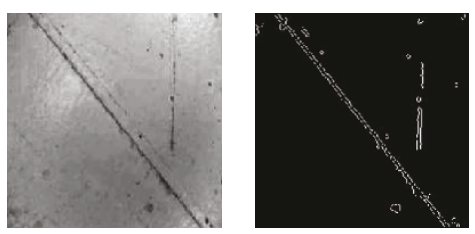

(a) With Canny: PCC surface

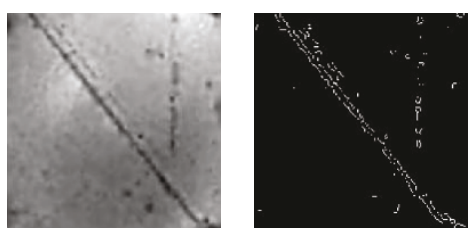

(b) With BEMD/Sobel: PCC surface

Figure 9

and 5 for asphalt and Figures 6 and 8 for PCC, both methods generally give acceptable results; BEMD/Sobel actually gives less noisy outputs, though, which is better.

Results for both methods were significantly more comparable for PCC surfaces. With the exception of Figure 7, the BEMD plus Sobel method matched the Canny method in the quality of detection. The BEMD is a local analysis method, so the expectation is a better performance than the Gaussian filter, which is a global analysis; fewer artifacts are expected with BEMD. However, the Sobel method still suffers from the effects of noise in an image even after smoothing with BEMD when the image has a lot of irregularities, as is the case for asphalt concrete surfaces.

\section{CONCLUSION}

The present paper is an exploration into the possible application of BEMD to image smoothing before crack detection with the Sobel edge detector; the results are compared with that of the Canny edge detector. Asphalt concrete and PCC images, both with cracks and without cracks, are analyzed and compared qualitatively. It is observed that although BEMD does well smoothing an image before edge detection with the Sobel method, the Sobel method still suffers from the effects of noise when the images have lots of irregularities present, as is the case for asphalt concrete surfaces. For images with less irregularities, such as the PCC surfaces, crack detection is more effective, and easily comparable to results from the Canny method; for PCC surfaces with no cracks, the BEMD/Sobel method gives outputs with less noise, which is better. Overall, the Canny edge detector performed better than the BEMD/Sobel method for asphalt surfaces, and slightly better for PCC surfaces. More research is needed to further explore the effectiveness of BEMD as a smoothing filter for quality crack detection.

\section{ACKNOWLEDGMENT}

Part of this paper has been presented at the SPIE Defense \& Security Symposium, Orlando, Florida, USA, 9-13 April 2007.

\section{REFERENCES}

[1] H. D. Cheng, J.-R. Chen, C. Glazier, and Y. G. Hu, "Novel approach to pavement cracking detection based on fuzzy set theory," Journal of Computing in Civil Engineering, vol. 13, no. 4, pp. 270-280, 1999.

[2] B. J. Lee and H. D. Lee, "Position-invariant neural network for digital pavement crack analysis," Computer-Aided Civil and Infrastructure Engineering, vol. 19, no. 2, pp. 105-118, 2004.

[3] P. Delagnes and D. Barba, "A markov random field for rectilinear structure extraction in pavement distress image analysis," in Proceedings of IEEE International Conference on Image Processing (ICIP '95), vol. 1, pp. 446-449, Washington, DC, USA, October 1995.

[4] C. Chandan, K. Sivakumar, E. Masad, and T. Fletcher, "Application of imaging techniques to geometry analysis of aggregate particles," Journal of Computing in Civil Engineering, vol. 18 , no. 1, pp. 75-82, 2004.

[5] J. M. Brzezicki and J. Kasperkiewicz, "Automatic image analysis in evaluation of aggregate shape," Journal of Computing in Civil Engineering, vol. 13, no. 2, pp. 123-128, 1999.

[6] L. Wang, X. Wang, L. Mohammad, and C. Abadie, "Unified method to quantify aggregate shape angularity and texture using Fourier analysis," Journal of Materials in Civil Engineering, vol. 17, no. 5, pp. 498-504, 2005.

[7] N. E. Huang, Z. Shen, S. R. Long, et al., "The empirical mode decomposition and the Hilbert spectrum for nonlinear and non-stationary time series analysis," Proceedings of the Royal Society A, vol. 454, no. 1971, pp. 903-995, 1998.

[8] N. E. Huang, M.-L. C. Wu, S. R. Long, et al., "A confidence limit for the empirical mode decomposition and Hilbert spectral analysis," Proceedings of the Royal Society A, vol. 459, no. 2037, pp. 2317-2345, 2003.

[9] A. Linderhed, "2-D empirical mode decompositions in the spirit of image compression," in Wavelet and Independent Component Analysis Applications IX, vol. 4738 of Proceedings of SPIE, pp. 1-8, Orlando, Fla, USA, April 2002.

[10] A. Linderhed, "Variable sampling of the empirical mode decomposition of two-dimensional signals," International Journal of Wavelets, Multiresolution and Information Processing, vol. 3, no. 3, pp. 435-452, 2005. 
[11] J. C. Nunes, Y. Bouaoune, E. Deléchelle, O. Niang, and Ph. Bunel, "Image analysis by bidimensional empirical mode decomposition," Image and Vision Computing, vol. 21, no. 12, pp. 1019-1026, 2003.

[12] J. C. Nunes, S. Guyot, and E. Deléchelle, “Texture analysis based on local analysis of the bidimensional empirical mode decomposition," Machine Vision and Applications, vol. 16, no. 3, pp. 177-188, 2005.

[13] A. Linderhed, "Image compression based on empirical mode decomposition," in Proceedings of the 3rd International Conference on Image and Graphics, pp. 430-443, Hong Kong, December 2004.

[14] S. Sinclair and G. G. S. Pegram, "Empirical mode decomposition in 2-D space and time: a tool for space-time rainfall analysis and nowcasting," Hydrology and Earth System Sciences Discussions, vol. 2, no. 1, pp. 289-318, 2005.

[15] L. Ding and A. Goshtasby, "On the Canny edge detector," Pattern Recognition, vol. 34, no. 3, pp. 721-725, 2001.

[16] P. Flandrin, G. Rilling, and P. Gonçalvés, "Empirical mode decomposition as a filter bank," IEEE Signal Processing Letters, vol. 11, no. 2, pp. 112-114, 2004.

[17] Z. Wu and N. E. Huang, "A study of the characteristics of white noise using the empirical mode decomposition method," Proceedings of the Royal Society A, vol. 460, no. 2046, pp. 15971611, 2004 